\title{
Estrategias Diagnósticas para exclusión o confirmación del Tromboembolismo de Pulmón
}

\begin{abstract}
Objetivo
Evaluar la eficacia de distintas estrategias empleadas en el diagnóstico del tromboembolismo de pulmón (TEP).
\end{abstract}

\section{Fuente de Datos}

Búsqueda de estudios en Medline, Embase, Pascal Biomed entre 1990 y 2003

\section{Selección de estudios y extracción de datos}

Se seleccionaron 48 estudios (11004 pacientes) que evaluaron distintas estrategias diagnósticas para la confirmación o exclusión de TEP, tomando como test de referencia la Angiografía pulmonar (AP) para la confirmación o la AP y/o el seguimiento clínico a tres meses, para la exclusión del diagnóstico. Se consideró que una estrategia diagnóstica posee una certeza diagnóstica suficiente cuando la probabilidad post test es superior al $85 \%$ para los test confirmatorios y menor del $5 \%$ para los test que excluyen el diagnóstico.

\section{Resultados Principales}

Las estrategias diagnósticas confirmatorias como de exclusión de TEP, la cantidad de estudios, pacientes, así como el coeficiente de probabilidad $(\mathrm{CP})^{*}$ con su intervalo de confianza se resumen en las tablas 1 y 2 .

Tabla 1. Estrategias confirmatorias de TEP

\begin{tabular}{l|c|c|c|c} 
& $\begin{array}{c}\text { Gantidad de } \\
\text { Estudios }\end{array}$ & $\begin{array}{c}\text { No. } \\
\text { depacientes }\end{array}$ & GP (t) $^{*}$ & (IC 95\%) \\
\hline V/Q alta probabilidad & 1 & 881 & 18,3 & 10,3 a 32,5 \\
\hline Perfusión pulmonar compatible con TEP & 1 & 390 & 7,1 & 4,6 a 11,0 \\
\hline TAC helicoidal + & 6 & 431 & 24,1 & 12,4 a 46,7 \\
\hline Eco doppler miembros inferiores (MMI) + & 4 & 378 & 16,2 & 5,6 a 46,7 \\
\hline Ecocardiograma & 2 & 148 & 5,0 & 2,3 a 10,6 \\
\hline RMI (**) & 5 & 221 & 11,7 & 3,6 a 37,8 \\
\hline
\end{tabular}

Tabla 2. Estrategias de exclusión de TEP.

\begin{tabular}{|c|c|c|c|c|}
\hline Estrategias de exclusión & $\begin{array}{c}\text { Cantidad de } \\
\text { Estudios }\end{array}$ & $\begin{array}{c}\text { No. } \\
\text { depacientes }\end{array}$ & CP $(-)^{*}$ & (IC 95\%) \\
\hline V/Q normal o casi normal $\left({ }^{* *}\right)$ & 9 & 3170 & 0,05 & 0,03 a 0,10 \\
\hline V/Q baja probababilidad & 1 & 881 & 0,36 & 0,25 a 0,50 \\
\hline Pertusión pulmonar No compatible c/IEP & 1 & 390 & 0,09 & 0,06 a 0,15 \\
\hline TAC helicoidal negativa $(* *)$ & 9 & 1197 & 0,11 & 0,06 a 0,19 \\
\hline TAC helicoidal neg. + Eco MMII. negativo & 3 & 1863 & 0,04 & 0,03 a 0,06 \\
\hline Eco doppler MMII. Negativo $\left({ }^{* *}\right)$ & 6 & 731 & 0,67 & 0,50 a 0,89 \\
\hline Eco cardiograma & 2 & 148 & 0,59 & 0,41 a 0,86 \\
\hline RMI & 5 & 221 & 0,20 & 012 a 0,34 \\
\hline Dimero D ElisA < 500 ugl $(* *)$ & 11 & 3368 & 0,08 & 0,04 a 0,18 \\
\hline Dimero D latex $<500$ ugl $(*)$ & 3 & 596 & 0,20 & 0,10 a 0,39 \\
\hline Dimero D latex semicuantitativo $\left({ }^{\star *}\right)$ & 2 & 201 & 0,29 & 0,03 a 0,46 \\
\hline Dimero D hemaglutinación negativo & 3 & 791 & 0,31 & 0.18 a 0.56 \\
\hline
\end{tabular}

$\left({ }^{\star \star}\right)$ Heterogeneidad de calidad de estudios y de resultados.

\section{Conclusiones}

Las estrategias diagnósticas que permiten confirmar el diagnóstico en las categorías de alta y media probabilidad clínica son el centellograma V/Q de alta probabilidad, la TAC helicoidal positiva, y el eco doppler de MMII positivo. En pacientes con baja a moderada probabilidad clínica los test que permiten excluir el diagnóstico son el centello V/Q normal, la TAC helicoidal negativa más eco doppler de MMII negativo, y el dímero D menor a $500 \mathrm{ug} / \mathrm{l}$ por ELISA. La TAC helicoidal sola, el V/Q de baja probabilidad, la angiorresonancia, y el dímero $\mathrm{D}$ por látex, tienen un peor $\mathrm{CP}^{*}$ negativo, pudiendo tan sólo descartar TEP si la probabilidad clínica es baja. Ningún test descarta TEP si la probabilidad clínica es alta, así como ninguno lo confirma si la probabilidad clínica es baja, todos estos pacientes deberán ir a la angiografía de pulmón.

Fuente de financiamiento: ninguna

\section{Comentario}

Existe un antes y un después en el diagnóstico de TEP con respecto a la publicación del PIOPED ${ }^{1}$ en 1990. El mismo trajo a conocimiento la relación entre la probabilidad clínica y centrellografica con respecto al diagnóstico final de TEP por angiografía. No obstante, si bien sentó las bases para el manejo diagnóstico posterior durante una década, el $65 \%$ de los pacientes caían en una categoría diagnóstica indefinida, requiriéndose llegar a la angiografía de pulmón, procedimiento con $0,5 \%$ de mortalidad y $5 \%$ de complicaciones.

Es así que en los últimos años surgen estrategias diagnósticas que intentan llegar al diagnóstico evitando la angiografía. Surgen así la TAC helicoidal, la angiorresonancia, el estudio doppler de MMII seriado, el dimero D, etc. Así como diversos algoritmos de diagnóstico basados en las distintas estrategias.

Existen grandes diferencias en la eficacia diagnóstica de los distintos test, y si bien toda revisión sistemática sobre estudios diagnósticos está dificultada por la heterogeneidad de los resultados entre los distintos estudios, las conclusiones del trabajo de Roy y col. son de una inestimable utilidad clínica. Los autores han expresado la perfomance de cada estrategia como coeficiente de probabilidad $(\mathrm{CP})^{\star}$, que indica la "magnitud del cambio" en la probabilidad de la enfermedad luego del resultado de un test. Los valores de corte de $85 \%$ y $5 \%$ para confirmación o exclusión del diagnóstico, si bien resultan arbitrarios y debatibles, ya fueron propuestos tiempo atrás por Kearon ${ }^{2}$ y gozan de aceptación general.

\section{Conclusiones del comentador}

En la práctica clínica actual el centello $V / Q$ y la TAC helicoidal compiten por la hegemonía diagnóstica del TEP. Según lo revisado por Roy y col. la perfomance diagnóstica puede ser equivalente, por tanto suelen ser otras variables las que determinan la utilización de uno u otro método (accesibilidad, experiencia del personal, presencia o no de infiltrado, derrame pleural, o severo enfisema en la radiografía de tórax, etc.). La probabilidad clínica se encuentra ampliamente estudiada por Wells ${ }^{3,4}$ y debidamente definida a través de puntajes, en donde, los síntomas clínicos de trombosis venosa profunda (TVP), la taquicardia mayor a 100 por minuto, la inmovilización o cirugía en el último mes, el antecedente de TVP/TEP previos, el cáncer y la hemoptisis, son las principales variables a tener en cuenta.

\section{Lucas Fernandez Otero [ Servicio de Clínica Médica. Hospital Italiano de Buenos Aires.}

Fernández Otero L. Estrategias Diagnósticas para exclusión o confirmación del Tromboembolismo de Pulmón. Evid Act Práct Ambul 2005;8:170. Disponible en URL: www.evidencia.org Comentado de: Roy PM, Colombet I, Durieux P et al. Systematic review and meta-analysis of strategies for the diagnosis of suspected pulmonary embolism. BMJ; 331: 259. PMID: 16052017

\section{Referencias}

1. Value of the ventilation/perfusion scan in acute pulmonary embolism. Results of the prospective investigation of pulmonary embolism diagnosis (PIOPED). The PIOPED investigators. JAMA 1990;263:2753-9.d

2. Diagnosis of pulmonary embolism. Kearon C. CMAJ 2003;168:183-94

3. Use of a clinical model for safe management of patients with suspected pulmonary embolism. Wells, PS, Ginsberg, JS, Anderson, DR, et. al. Ann Intern Med 1998; 129:997. 